\title{
Analysis and Design of Wide-Band SiGe HBT Active Mixers
}

\author{
Johansen, Tom Keinicke; Krozer, Viktor; Vidkjær, Jens
}

Published in:

I E E E Transactions on Microwave Theory and Techniques

Link to article, DOI:

10.1109/TMTT.2005.850421

Publication date:

2005

Document Version

Publisher's PDF, also known as Version of record

Link back to DTU Orbit

Citation (APA):

Johansen, T. K., Krozer, V., \& Vidkjær, J. (2005). Analysis and Design of Wide-Band SiGe HBT Active Mixers. I E E E Transactions on Microwave Theory and Techniques, 53(7), 2389-2397.

https://doi.org/10.1109/TMTT.2005.850421

\section{General rights}

Copyright and moral rights for the publications made accessible in the public portal are retained by the authors and/or other copyright owners and it is a condition of accessing publications that users recognise and abide by the legal requirements associated with these rights.

- Users may download and print one copy of any publication from the public portal for the purpose of private study or research.

- You may not further distribute the material or use it for any profit-making activity or commercial gain

- You may freely distribute the URL identifying the publication in the public portal

If you believe that this document breaches copyright please contact us providing details, and we will remove access to the work immediately and investigate your claim. 


\title{
Analysis and Design of Wide-Band SiGe HBT Active Mixers
}

\author{
Tom K. Johansen, Member, IEEE, Jens Vidkjær, Member, IEEE, and Viktor Krozer, Senior Member, IEEE
}

\begin{abstract}
The frequency response of SiGe HBT active mixers based on the Gilbert cell topology is analyzed theoretically. The time-varying operation of the active mixer is taken into account by applying conversion matrix analysis. The main bandwidth-limiting mechanisms experienced in SiGe HBT active mixers performing frequency conversion of wide-band signals is discussed. The analysis is verified by computer simulations using a realistic high-frequency large-signal SiGe HBT model. An active mixer design based on the Gilbert cell topology modified for wide-band operation using emitter degenerated transconductance stage and shunt feedback load stage is discussed. Experimental results are given for an active mixer implemented in a $0.8-\mu \mathrm{m}$ 35-GHz $f_{T}$ SiGe HBT BiCMOS process.
\end{abstract}

Index Terms-Active mixer circuits, frequency-response analysis, SiGe HBT.

\section{INTRODUCTION}

$\mathbf{R}$ ECENT advances in radar and wireless communication systems demand monolithic microwave integrated circuits (MMICs) providing frequency conversion, modulation, and demodulation of wide-band signals around microwave carriers. Active mixers based on the Gilbert cell topology [1] are usually preferred for monolithic integration of these functions due to their high conversion gain over a broad frequency band, and good port-to-port isolation. Good microwave performance has been reported for active mixers implemented in InP HBT [2], SiGe HBT [3], GaAs HBT [4], and GaAs pseudomorphic high electron-mobility transistor (pHEMT) [5] technologies.

Previously reported monolithic active mixers have mostly been optimized for downconversion performance in wireless applications. Typically, such mixers are capable of frequency conversion of narrow-band signals over a wide range of RF input frequencies to a fixed low IF output frequency. This differs from the demands on active mixers for wide-band applications. Here, the wide-band signal experiences bandwidth limitations at both the RF input frequency and IF output frequency. In order to optimize the performance of active mixers for wide-band applications, an understanding of the main bandwidth limitations in the Gilbert cell topology is needed. The large local-oscillator (LO) signal applied to the transistors in the switching stage of the Gilbert cell gives rise to a time-varying circuit. Therefore, a traditional linear time-invariant circuit analysis for finding the bandwidth limitations becomes invalid. Instead, a conversion

Manuscript received September 20, 2004; revised December 21, 2004. This work was supported by the Danish Technical Research Council.

The authors are with the Section of Electromagnetic Systems, Ørsted•DTU, Technical University of Denmark, 2800 Lyngby, Denmark.

Digital Object Identifier 10.1109/TMTT.2005.850421

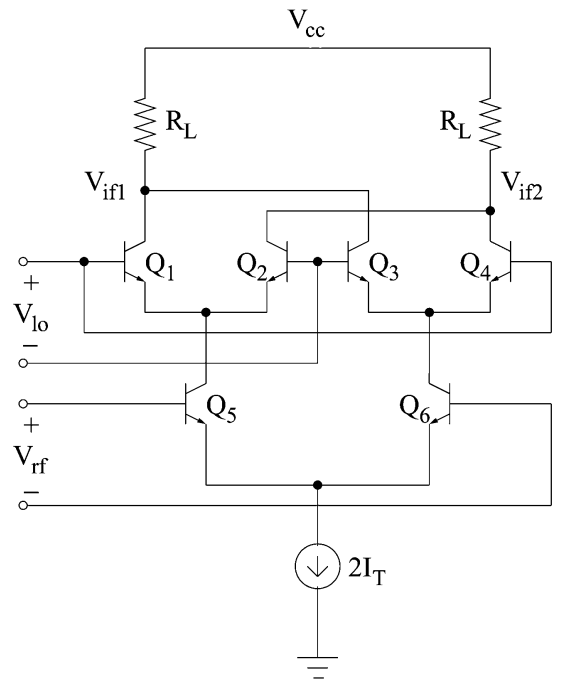

Fig. 1. Gilbert cell mixer.

matrix analysis [6] should be applied in order to find an expression for the conversion gain as a function of the RF input frequency and IF output frequency. A previous attempt [7] to analyze the conversion gain of the Gilbert cell neglected the IF output frequency response and, thus, only considered the downconversion of narrow-band signals.

This paper describes a theoretical analysis of the conversion gain of SiGe HBT active mixers based on the Gilbert cell topology. An analytical expression is derived, which is capable of predicting the main bandwidth limitations for active mixers performing frequency conversion of wide-band signals. The analysis is verified with harmonic-balance simulations using a realistic SiGe HBT high-frequency large-signal model. The insight gained from the analysis is used in the design optimization of an SiGe HBT active mixer for wide-band applications. Experimental results are given for an active mixer implemented in a $0.8-\mu \mathrm{m} 35-\mathrm{GHz} f_{T}$ SiGe HBT BiCMOS process.

\section{FREQUENCY-ReSPONSE ANALYsis}

The schematic representation of an SiGe HBT active mixer based on the Gilbert cell topology is shown in Fig. 1. The Gilbert cell mixer consists of the transconductance stage $\left(Q_{5}-Q_{6}\right)$, switching quad $\left(Q_{1}-Q_{4}\right)$, and load circuit. The circuit is biased from a tail current source $2 I_{T}$. The transconductance stage converts the input voltage signal at the RF frequency into a current signal. The switching quad $\left(Q_{1}-Q_{4}\right)$ then ideally multiplies this current by \pm 1 at the rate of the LO signal, thus enabling the wanted frequency conversion. The output current signal at the 


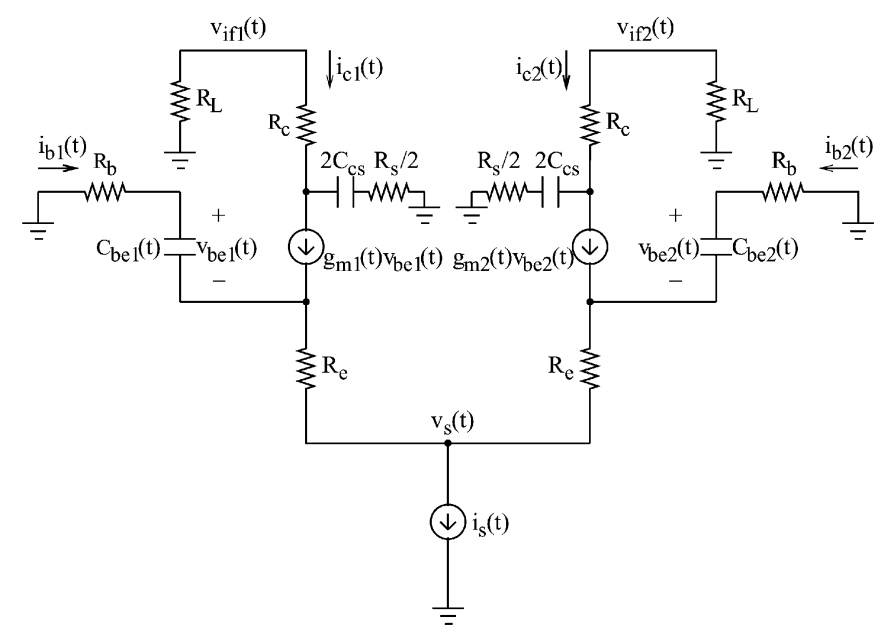

Fig. 2. Time-varying small-signal equivalent-circuit model.

IF frequency from the switching quad is finally converted into a voltage signal by the load circuit. Neglecting any device parasitics, the voltage conversion gain of the Gilbert cell mixer is given as [8]

$$
G_{\mathrm{cnv}}=\frac{2}{\pi} \frac{I_{T}}{V_{T}} R_{L}
$$

where $V_{T}$ is the thermal voltage. When the device parasitics are taken into account, the expression for the conversion gain (1) changes, as will be shown using conversion matrix analysis below. First of all, the conversion-gain expression becomes a function of two frequencies, i.e., the RF input frequency and IF output frequency. Secondly, the low-frequency conversion gain is reduced because of the presence of series resistances.

\section{A. Conversion Matrix Analysis}

The starting point for conversion matrix analysis is to make a linearization around a time-varying operation point for the nonlinear elements of the Gilbert cell mixer circuit. The large-signal LO drive pumps the transistors in the switching quad $\left(Q_{1}-Q_{4}\right)$. The common-emitter points for the differential transistor pairs $\left(Q_{1}-Q_{2}\right)$ and $\left(Q_{3}-Q_{4}\right)$ are virtual ground for the large-signal LO drive. As a consequence, the transconductance stage is time invariant under small-signal RF drive conditions. At high frequencies, the dominating nonlinearities in the switching quad transistors are associated with the transfer current and the base-emitter charge [9] so the simplified time-varying small-signal equivalent-circuit model shown in Fig. 2 is sufficient for analysis. In this model, only one pair of transistors in the switching quad is considered, which is justified by the symmetry of the switching quad. The base-collector capacitance and base-emitter conductance have been neglected because their contributions are small, but significantly complicate the analysis. Constant values are assumed for the base resistance $R_{b}$, emitter resistance $R_{e}$, and collector resistance $R_{c}$. A constant value equal to the bias value is also used for the collector-substrate capacitance $C_{\mathrm{CS}}$ and the

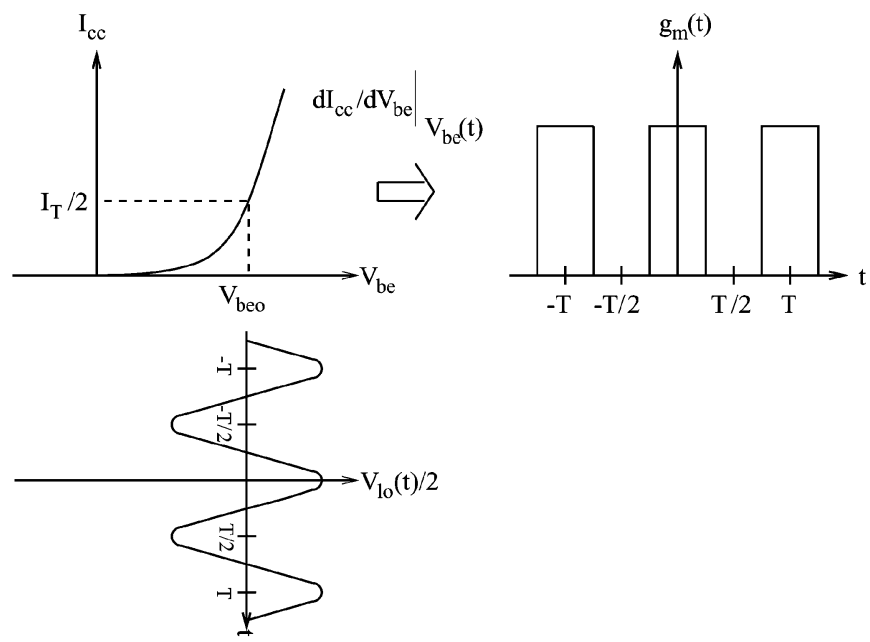

Fig. 3. Evaluation of time-varying transconductance.

substrate resistance $R_{s}$, respectively. Furthermore, the value of the collector-substrate capacitance is doubled and the value of the substrate resistance is halved in order to take into account the collector dotting in the switching quad. The time-varying switching stage is driven from an ideal small-signal current source $i_{s}(t)$. This current source can be determined from traditional linear time-invariant analysis of the transconductance stage.

The nonlinear $I-V$ characteristic of the transfer current $I_{\mathrm{cc}}$ results in a time-varying transconductance waveform given by

$$
g_{m}(t)=\left.\frac{d I_{\mathrm{cc}}}{d V_{\mathrm{be}}}\right|_{V_{\mathrm{be}}=\frac{V_{\mathrm{lo}}(t)}{2}+V_{\mathrm{beo}}}
$$

where $V_{\mathrm{lo}}(t)$ is the applied LO signal.

A graphical evaluation of the transconductance waveform $g_{m}(t)$ for the switching quad transistors under large-signal LO drive is illustrated in Fig. 3. Notice that the large-signal LO drive is superimposed on the base bias voltage $V_{\text {beo }}$. Assuming instantaneous switching of the transistors $\left(Q_{1}-Q_{4}\right)$, the transconductance waveform resembles a square wave given as

$$
\begin{aligned}
& g_{m}(t) \\
& \quad= \begin{cases}\frac{I_{T}}{V_{T}}, & \text { if }|t-n T| \leq T / 4 ; \quad n=0, \pm 1, \pm 2, \pm 3, \ldots \\
0, & \text { otherwise }\end{cases}
\end{aligned}
$$

where $T$ is the period of the LO signal. The instantaneous switching assumption remains valid as long as the LO amplitude across the internal junctions is sufficient to assure hard limiting of the differential pairs in the switching quad. Similarly, the nonlinear $Q-V$ characteristic of the base-emitter charge $Q_{\text {be }}$ results in a time-varying capacitance waveform given by

$$
C_{\mathrm{be}}(t)=\left.\frac{d Q_{\mathrm{be}}}{d V_{\mathrm{be}}}\right|_{V_{\mathrm{be}}=\frac{V_{\mathrm{lo}}(t)}{2}+V_{\mathrm{beo}}}
$$


and includes depletion and diffusion terms. Assuming instantaneous switching, the base-emitter capacitance waveform also resembles a square wave given as

$$
C_{\mathrm{be}}(t)= \begin{cases}\frac{\tau_{f} I_{T}}{V_{T}}+4 C_{\mathrm{jeo}}, & \text { if }|t-n T| \leq T / 4 \\ 0, \quad \text { otherwise } & n=0, \pm 1, \pm 2, \pm 3, \ldots\end{cases}
$$

where $\tau_{f}$ is the forward transit time and $C_{\text {jeo }}$ is the zero bias base-emitter depletion capacitance. The peak value of $4 C_{\mathrm{jeo}}$ is justified by the fact that the average value of the base-emitter depletion capacitance for a forward-biased junction is approximately $2 C_{\text {jeo }}$ [10]. This formulation provides a satisfactory tradeoff between analysis accuracy and easy interpretation of the final expression for the conversion gain.

The time-varying elements in the Gilbert cell mixer in the presence of the small-signal RF excitation give rise to mixing frequencies represented as [11]

$$
\omega_{n}=\omega_{\text {if }}+n \omega_{\mathrm{LO}}, \quad n=0, \pm 1, \pm 2, \pm 3, \ldots
$$

where $\omega_{\text {if }}=\left|\omega_{\mathrm{LO}}-\omega_{\mathrm{rf}}\right|$ is the IF angular frequency and $\omega_{\mathrm{rf}}$ and $\omega_{\mathrm{LO}}$ are the RF and LO angular frequencies, respectively. For the present investigation, only the response at the IF frequency, as well as the upper and lower RF sideband, are considered in the analysis.

Conversion matrices relate the current and voltage components at different mixing frequencies in a time-varying circuit. For the time-varying transconductance, the relation is

$$
\left[\begin{array}{c}
I_{-1}^{*} \\
I_{0} \\
I_{1}
\end{array}\right]=\left[\begin{array}{ccc}
G_{0} & G_{-1} & G_{-2} \\
G_{1} & G_{0} & G_{-1} \\
G_{2} & G_{1} & G_{0}
\end{array}\right]\left[\begin{array}{c}
V_{-1}^{*} \\
V_{0} \\
V_{1}
\end{array}\right]
$$

where $I_{n}$ are the resulting currents at the mixing frequency $\omega_{n}$ for voltages $V_{m}$ at all relevant excitations frequencies $\omega_{m}$, and the asterisks denote a complex conjugate. The elements of the conversion matrix are the Fourier coefficients of the timevarying transconductance given as

$$
G_{n}=\left\{\begin{array}{l}
\frac{I_{T}}{2 V_{T}}, \quad \text { for } n=0 \\
\frac{I_{T}}{\pi V_{T}}, \quad \text { for } n= \pm 1 \\
0, \quad \text { for } n= \pm 2
\end{array}\right.
$$

where $G_{0}$ corresponds to the average transconductance. Similar for the time-varying base-emitter capacitance, the relation is

$$
\begin{array}{r}
{\left[\begin{array}{c}
I_{-1}^{*} \\
I_{0} \\
I_{1}
\end{array}\right]=\left[\begin{array}{ccc}
j \omega_{-1} & 0 & 0 \\
0 & j \omega_{0} & 0 \\
0 & 0 & j \omega_{1}
\end{array}\right]\left[\begin{array}{ccc}
C_{0} & C_{-1} & C_{-2} \\
C_{1} & C_{0} & C_{-1} \\
C_{2} & C_{1} & C_{0}
\end{array}\right]} \\
\\
\times \\
\times \\
\end{array}
$$

where the diagonal matrix containing the mixing frequencies is the frequency-domain equivalent to a time-domain differentiation. The Fourier coefficients for the time-varying base-emitter capacitance are given as

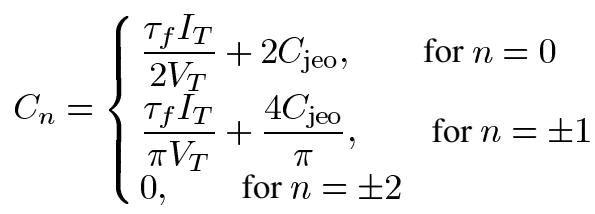

where $C_{0}$ corresponds to the average base-emitter capacitance.

Kirchoff's laws must hold for the individual current and voltage components in the vectors in (7) and (9). As a consequence, the time-varying equivalent circuit for the Gilbert cell mixer in Fig. 2 can thus be analyzed in a manner analogous to that used for linear time-invariant circuit. An important difference is that all elements must have a conversion matrix form. For fixed-value components, this form is a diagonal matrix, and the element value must occupy all the locations on the main diagonal [6]. Kirchoff's voltage law makes it possible to express the base-emitter voltage vectors $\mathbf{V}_{\text {be } \mathbf{1}}$ and $\mathbf{V}_{\text {be } \mathbf{2}}$ in terms of the voltage vector $\mathbf{V}_{s}$ at the common-emitter point as

$$
\begin{aligned}
& \mathbf{V}_{\mathbf{b e} 1}=-\left[\mathbf{I}+\mathbf{G}_{\mathbf{m} \mathbf{1}} \mathbf{R}_{\mathbf{e}}+j \Omega \mathbf{C}_{\mathrm{be} \mathbf{1}}\left(\mathbf{R}_{\mathbf{b}}+\mathbf{R}_{\mathbf{e}}\right)\right]^{-1} \mathbf{V}_{\mathbf{s}} \\
& \mathbf{V}_{\mathbf{b e} 2}=-\left[\mathbf{I}+\mathbf{G}_{\mathbf{m} 2} \mathbf{R}_{\mathbf{e}}+j \Omega \mathbf{C}_{\mathbf{b e} 2}\left(\mathbf{R}_{\mathbf{b}}+\mathbf{R}_{\mathbf{e}}\right)\right]^{-1} \mathbf{V}_{\mathbf{s}}
\end{aligned}
$$

where $\mathbf{I}$ is the identity matrix, $\mathbf{G}_{\mathbf{m} \mathbf{1}}$ is the conversion matrix for $g_{m 1}(t), \mathbf{G}_{\mathbf{m} 2}$ is the conversion matrix for $g_{m 2}(t), \mathbf{C}_{\mathbf{b e} \mathbf{1}}$ is the conversion matrix for $C_{\mathrm{be} 1}(t), \mathbf{C}_{\mathrm{be} 2}$ is the conversion matrix for $C_{\mathrm{be} 2}(t), \Omega$ is the diagonal matrix containing the mixing frequencies as described above, $\mathbf{R}_{\mathrm{e}}$ is a diagonal matrix with elements $R_{e}$, and $\mathbf{R}_{\mathrm{b}}$ is a diagonal matrix with elements $R_{b}$. Kirchoff's current law gives

$$
\left(\mathbf{G}_{\mathbf{m} 1}+j \Omega \mathbf{C}_{\mathbf{b e} 1}\right) \mathbf{V}_{\mathbf{b e} 1}+\left(\mathbf{G}_{\mathbf{m} 2}+j \Omega \mathbf{C}_{\mathbf{b e} 2}\right) \mathbf{V}_{\mathbf{b e} 2}=\mathbf{I}_{\mathbf{s}}
$$

where $\mathbf{I}_{\mathbf{S}}$ is the small-signal excitation vector with typically only one nonzero element. Substitution of (11) and (12) into (13) determines $\mathbf{V}_{\mathbf{S}}$ in terms of the current source excitation $\mathbf{I}_{\mathbf{S}}$ as

$$
\begin{aligned}
& \mathbf{V}_{\mathbf{s}}=-\left[( \mathbf { G } _ { \mathbf { m } \mathbf { 1 } } + j \Omega \mathbf { C } _ { \mathrm { be } \mathbf { 1 } } ) \left[\mathbf{I}+\mathbf{G}_{\mathbf{m} \mathbf{1}} \mathbf{R}_{\mathbf{e}}\right.\right. \\
& \left.+j \Omega \mathbf{C}_{\mathbf{b e 1}}\left(\mathbf{R}_{\mathbf{b}}+\mathbf{R}_{\mathbf{e}}\right)\right]^{-1} \\
& +\left(\mathbf{G}_{\mathbf{m} 2}+j \boldsymbol{\Omega} \mathbf{C}_{\mathbf{b e}, 2}\right)\left[\mathbf{I}+\mathbf{G}_{\mathbf{m} 2} \mathbf{R}_{\mathbf{e}}\right. \\
& \left.\left.+j \Omega \mathbf{C}_{\mathrm{be} 2}\left(\mathbf{R}_{\mathrm{b}}+\mathbf{R}_{\mathbf{e}}\right)\right]^{-1}\right]^{-1} \mathbf{I}_{\mathbf{s}} .
\end{aligned}
$$

The differential output voltage vector $\mathbf{V}_{\text {if2 }}-\mathbf{V}_{\text {if1 }}$ can be expressed in terms of the base-emitter voltage vectors $\mathbf{V}_{\mathbf{b e 1}}$ and $\mathbf{V}_{\text {be2 }}$ as

$$
\begin{aligned}
\mathbf{V}_{\mathbf{i f 2}}-\mathbf{V}_{\text {if1 }}=[\mathbf{I}+2 & \left.\mathbf{Y}_{\mathbf{s}}\left(\mathbf{R}_{\mathbf{c}}+\mathbf{R}_{\mathbf{L}}\right)\right]^{-1} \\
& \times\left[\mathbf{G}_{\mathbf{m} \mathbf{1}} \mathbf{V}_{\mathbf{b e} 1}-\mathbf{G}_{\mathrm{m} 2} \mathbf{V}_{\mathbf{b e} 2}\right] \mathbf{R}_{\mathbf{L}}
\end{aligned}
$$


where $\mathbf{Y}_{\mathbf{s}}$ is a diagonal matrix with frequency-dependent elements given as

$$
\mathbf{Y}_{\mathbf{s}}=\left[\begin{array}{ccc}
\frac{j \omega_{-1} C_{\mathrm{cs}}}{1+j \omega_{-1} R_{s} C_{\mathrm{cs}}} & 0 & 0 \\
0 & \frac{j \omega_{0} C_{\mathrm{cs}}}{1+j \omega_{0} R_{s} C_{\mathrm{cs}}} & 0 \\
0 & 0 & \frac{j \omega_{1} C_{\mathrm{cs}}}{1+j \omega_{1} R_{\mathrm{s}} C_{\mathrm{cs}}}
\end{array}\right]
$$

$\mathbf{R}_{\mathrm{c}}$ is a diagonal matrix with elements $R_{c}$, and $\mathbf{R}_{\mathbf{L}}$ is a diagonal matrix with elements $R_{L}$. Combining expressions (11), (12), (14), and (15), the conversion switching gain $G_{\mathrm{sw}}\left(\omega_{\mathrm{if}}, \omega_{\mathrm{rf}}\right)$ defined as the ratio between the differential output voltage at the IF frequency and the ideal current excitation at the RF frequency can be determined. As expected for a balanced structure, the RF excitation is fully suppressed in the differential output voltage. Substitution of the Fourier coefficients for the time-varying transconductance and time-varying base-emitter capacitance into the expression for the conversion switching gain, and simplifying using a symbolic mathematical software tool, gives

$$
G_{\mathrm{sw}}\left(\omega_{\mathrm{rf}}, \omega_{\mathrm{if}}\right)=\frac{G_{\mathrm{sw} 0}\left(1-j \frac{\omega_{\mathrm{if}}}{z_{1}}\right)}{\left(1-j \frac{\omega_{\mathrm{if}}}{p_{1}}\right)\left(1-j \frac{\omega_{\mathrm{if}}}{p_{2}}\right)\left(1-j \frac{\omega_{\mathrm{rf}}}{p_{3}}\right)}
$$

where the low-frequency conversion gain $G_{\mathrm{sw} 0}$ is given as

$$
G_{\mathrm{Sw} 0}=\frac{2}{\pi} \frac{R_{L}}{1+\frac{\left(-8+\pi^{2}\right)}{2 \pi^{2}} \frac{I_{T}}{V_{T}} R_{e}}
$$

and shows a very small gain reduction due to the emitter resistances in the switching quad transistors. The conversion switching gain shows a two-pole and one zero response with respect to the IF frequency expressed as

$$
\frac{1}{p_{1}}=-\frac{\frac{\tau_{f} I_{T}}{V_{T}}+4 C_{\text {jeo }}}{1+\frac{\left(-8+\pi^{2}\right)}{2 \pi^{2}} \frac{I_{T}}{V_{T}} R_{e}} \frac{\left(-8+\pi^{2}\right)}{2 \pi^{2}}\left(R_{b}+R_{e}\right)
$$

and

$$
\frac{1}{p_{2}}=-C_{\mathrm{cs}}\left(R_{s}+2 R_{c}+2 R_{L}\right)
$$

for the poles and

$$
\frac{1}{z_{1}}=-C_{\mathrm{cs}} R_{s}
$$

for the zero, respectively. It is observed that a significant substrate resistance $R_{s}$ actually may lead to partial pole-zero cancellation in the IF frequency response of the Gilbert cell mixer. The single pole response with respect to the RF frequency is expressed as

$$
\frac{1}{p_{3}}=-\left(\tau_{f}+\frac{4 C_{\mathrm{jeo}} V_{T}}{I_{T}}\right)
$$

and represent the finite current switching delay in the quad transistors. The gain $G_{\mathrm{tr}}\left(\omega_{\mathrm{rf}}\right)$ for the transconductance stage defined as the ratio between the short-circuited output current and the RF voltage excitation is easily determined from a linear time-invariant analysis of a small-signal hybrid- $\pi$ equivalent circuit with the following approximate result [12]:

$$
G_{\mathrm{tr}}\left(\omega_{\mathrm{rf}}\right)=\frac{G_{\mathrm{tr} 0}}{\left(1-j \frac{\omega_{\mathrm{rf}}}{p 4}\right)}
$$

where the low-frequency transconductance $G_{\operatorname{tr} 0}$ is given as

$$
G_{\text {tr } 0}=\frac{g_{m 5}}{1+R_{e 5}\left(g_{m 5}+g_{\mathrm{be} 5}\right)+R_{b 5} g_{\mathrm{be} 5}}
$$

and where the elements refer to $Q_{5}$ in Fig. $1 .{ }^{1}$ It shows a reduction in the low-frequency transconductance due to the series resistances $R_{e 5}$ and $R_{b 5}$. The frequency response of the transconductance stage is determined by a single-pole response given as

$$
\frac{1}{p 4} \approx-\frac{\left(R_{b 5}+R_{e 5}\right) g_{m 5}}{2 \pi f_{T}\left(1+R_{e 5}\left(g_{m 5}+g_{\mathrm{be} 5}\right)+R_{b 5} g_{\mathrm{be} 5}\right)}
$$

where the influence of the base-collector capacitance, collector-substrate capacitance, and collector resistance have been neglected. This is justified by the fact that, at any instant, the switching quad presents a low impedance to the transconductance stage. The final expression for the voltage conversion gain of the Gilbert cell mixer $G_{\text {cnv }}\left(\omega_{\mathrm{rf}}, \omega_{\mathrm{if}}\right)$ is then found simply by multiplying (17) and (23) as follows:

$$
\begin{aligned}
& G_{\mathrm{cnv}}\left(\omega_{\mathrm{rf}}, \omega_{\mathrm{if}}\right) \\
& =\frac{G_{\mathrm{tr} 0} G_{\mathrm{sw} 0}\left(1-j \frac{\omega_{\mathrm{if}}}{z_{1}}\right)}{\left(1-j \frac{\omega_{\mathrm{if}}}{p_{1}}\right)\left(1-j \frac{\omega_{\mathrm{if}}}{p_{2}}\right)\left(1-j \frac{\omega_{\mathrm{rf}}}{p_{3}}\right)\left(1-j \frac{\omega_{\mathrm{rf}}}{p_{4}}\right)}
\end{aligned}
$$

and shows a two-pole response with respect to the RF frequency and a two-pole and one zero response with respect to the IF frequency.

\section{B. Analysis Verification}

To verify the derived expression for the conversion gain, harmonic-balance simulations on a Gilbert cell mixer were performed using Agilent ADS. The high-frequency large-signal model for the SiGe HBT devices used in the simulations is shown in Fig. 4. This model includes the dominating nonlinearities and parasitic elements in the SiGe HBT devices [13]. The large-signal model parameters for a $4 \times 0.8 \mu \mathrm{m}^{2}$ area SiGe HBT device used in the current investigation are $I_{s}=6.3 \mathrm{aA}$, $\beta_{f}=80, \tau_{f}=3.6 \mathrm{pS}, C_{\mathrm{jeo}}=25.1 \mathrm{fF}, C_{\mathrm{jco}}=6.8 \mathrm{fF}, X_{\mathrm{cjc}}=$ $0.4, C_{\mathrm{jso}}=37.7 \mathrm{fF}, R_{b}=114.0 \Omega, R_{e}=3.0 \Omega, R_{c}=12.0 \Omega$, and $R_{s}=965.0 \Omega$. Several of the large-signal model parameters has been extracted from a dedicated small-signal equivalent-circuit model using the method described in [14].

The Gilbert cell mixer is designed for a low-frequency conversion gain of $20 \mathrm{~dB}$ at a bias current of $2 I_{T}=2.8 \mathrm{~mA}$.

\footnotetext{
${ }^{1} \mathrm{~A}$ differential-mode half-circuit is considered here.
} 


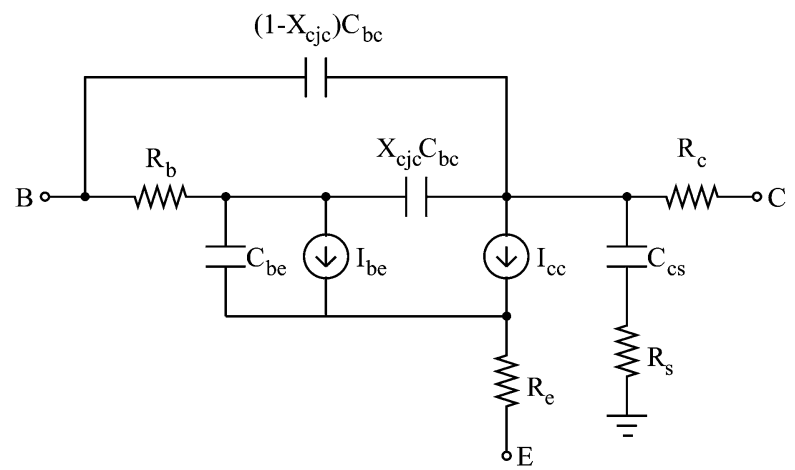

Fig. 4. High-frequency large-signal SiGe HBT model for harmonic-balance simulations.

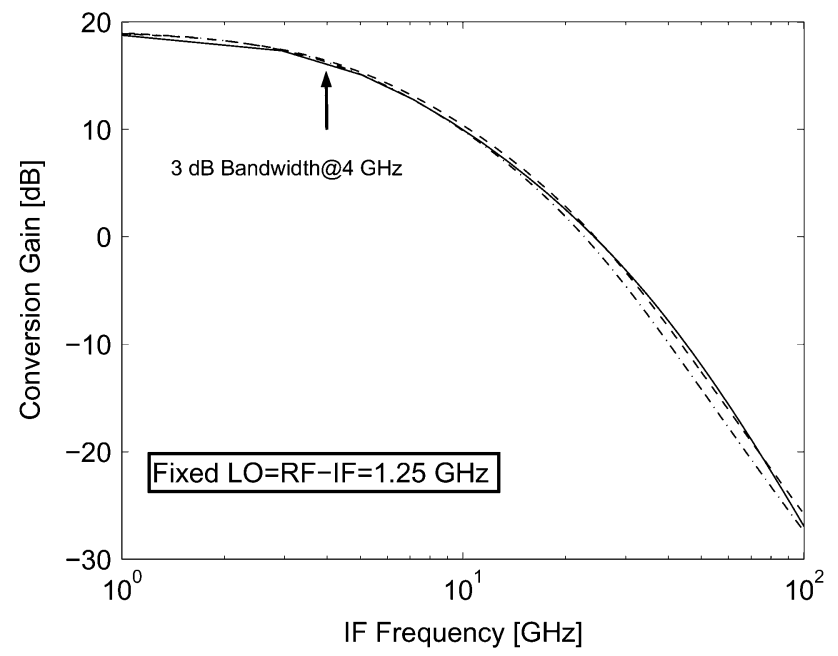

Fig. 5. Comparing calculated and simulated conversion gain for the Gilbert cell mixer. Solid line: calculated. Dashes line: simulated without $C_{\mathrm{bc}}$. Dotted-dashed line: simulated with $C_{\mathrm{bc}}$.

The SiGe HBT devices in the transconductance stage and the switching quad each have an emitter area of $4 \times 0.8 \mu \mathrm{m}^{2}$. The devices in the transconductance stage are biased at peak $f_{T}$. Fig. 5 compares the calculated conversion gain versus IF frequency for a fixed LO frequency of $1.25 \mathrm{GHz}$ with harmonicbalance simulations. An excellent agreement is observed for both the 3-dB IF bandwidth and the high-frequency rolloff rate up to $100 \mathrm{GHz}$. As shown in Fig. 5, the effect of the base-collector capacitance is small, and neglecting it in the analysis is justified.

\section{Discussion}

It is instructive to investigate the main bandwidth limitations in the Gilbert cell mixer. The expression derived for the conversion gain provides improved design insight into the optimization of Gilbert cell mixers for wide-band applications. In Table I, the individual contributions from the poles $\left(p_{1}-p_{4}\right)$ and the zero $\left(z_{1}\right)$ to the 3-dB IF bandwidth is calculated under the assumption of fixed LO frequency of $1.25 \mathrm{GHz}$. The dominant poles are related to the transconductance stage and the output loading at the switching quad. As mentioned previously, the zero due to the substrate resistance partly compensates for the dominant
TABLE I

INDIVIDUAL CONTRIBUTION FROM POLES AND ZEROS TO 3-dB IF BANDWIDTH OF SiGe HBT GILBERT CELl MiXER

\begin{tabular}{c|c}
\hline Contribution & IF Bandwidth $[\mathrm{GHz}]$ \\
\hline$p_{1}$ & 49.7 \\
\hline$p_{2}$ & 5.8 \\
\hline$p_{3}$ & 28.5 \\
\hline$p_{4}$ & 5.8 \\
\hline$z_{1}$ & 9.9 \\
\hline
\end{tabular}

pole due to output loading at the switching quad. If a lower bias current is used, the pole $\left(p_{3}\right)$ due to the finite current switching delay in the quad transistors may become significant.

\section{Wide-BAND Active MiXer Design}

The schematic of an SiGe HBT active mixer design based on a Gilbert cell topology modified for wide-band operation is shown in Fig. 6. The Gilbert cell employs emitter degeneration $\left(R_{e}\right.$ and $\left.C_{e}\right)$ for the transconductance stage $\left(Q_{5}-Q_{6}\right)$ and a differential amplifier stage $\left(Q_{7}-Q_{8}\right)$ with resistive shunt feedback $\left(R_{f}\right)$ for the load circuit. The Gilbert cell core is embedded with emitter follower stages $\left(Q_{13}-Q_{16}\right)$ with on-chip 50- $\Omega$ shunt resistors for input buffering at the RF and LO ports, as well as emitter follower stages $\left(Q_{9}-Q_{12}\right)$ for output buffering at the IF port. Two stages of emitter followers are needed at the IF port in order to minimize loading of the shunt feedback stage. Resistive emitter degeneration in the transconductance stage allows a significant extension of the RF bandwidth. This is, however, at the expense of reduced conversion gain, as shown in (23)-(25). A peaking capacitor provides a zero in the transfer function, which extends the transconductance stage bandwidth with relaxed resistive emitter degeneration, as shown by the following expression [15]:

$$
\begin{aligned}
G_{\mathrm{tr}}\left(\omega_{\mathrm{rf}}\right) & =\frac{G_{\mathrm{tr} 0}\left(1-j \frac{\omega_{\mathrm{rf}}}{z_{1 e}}\right)}{1+j \omega_{\mathrm{rf}} \frac{2 K_{e}}{\omega_{0 e}}-\frac{\omega_{\mathrm{rf}}^{2}}{\omega_{0 e}^{2}}} \\
G_{\mathrm{tr} 0} & \approx \frac{g_{m 5}}{1+g_{m 5} R_{e} / 2} \\
\frac{1}{z_{1 e}} & =-C_{e} R_{e} \\
\omega_{0 e} & \approx \sqrt{\frac{1+g_{m 5} R_{e} / 2}{R_{b 5} C_{\mathrm{be} 5} C_{e} R_{e}}} \\
K_{e} & \approx \frac{1}{2} \frac{C_{e} R_{e}+C_{\mathrm{be} 5}\left(R_{b 5}+R_{e} / 2\right)}{\sqrt{\left(1+g_{m 5} R_{e} / 2\right) R_{b 5} C_{\mathrm{be} 5} C_{e} R_{e}}}
\end{aligned}
$$

where the approximation $\left(R_{b 5}+R_{e} / 2\right) \ll R_{\mathrm{be} 5}$ is applied. It should be mentioned, however, that the combination of resistive and capacitive emitter degeneration leads to nonoptimum linearity and noise for the active mixer compared to inductive emitter degeneration typically found in narrow-band designs [8]. The shunt feedback load circuit originally described in [16] has several advantages compared with other methods for extending the IF bandwidth of the Gilbert cell mixer [17]. 


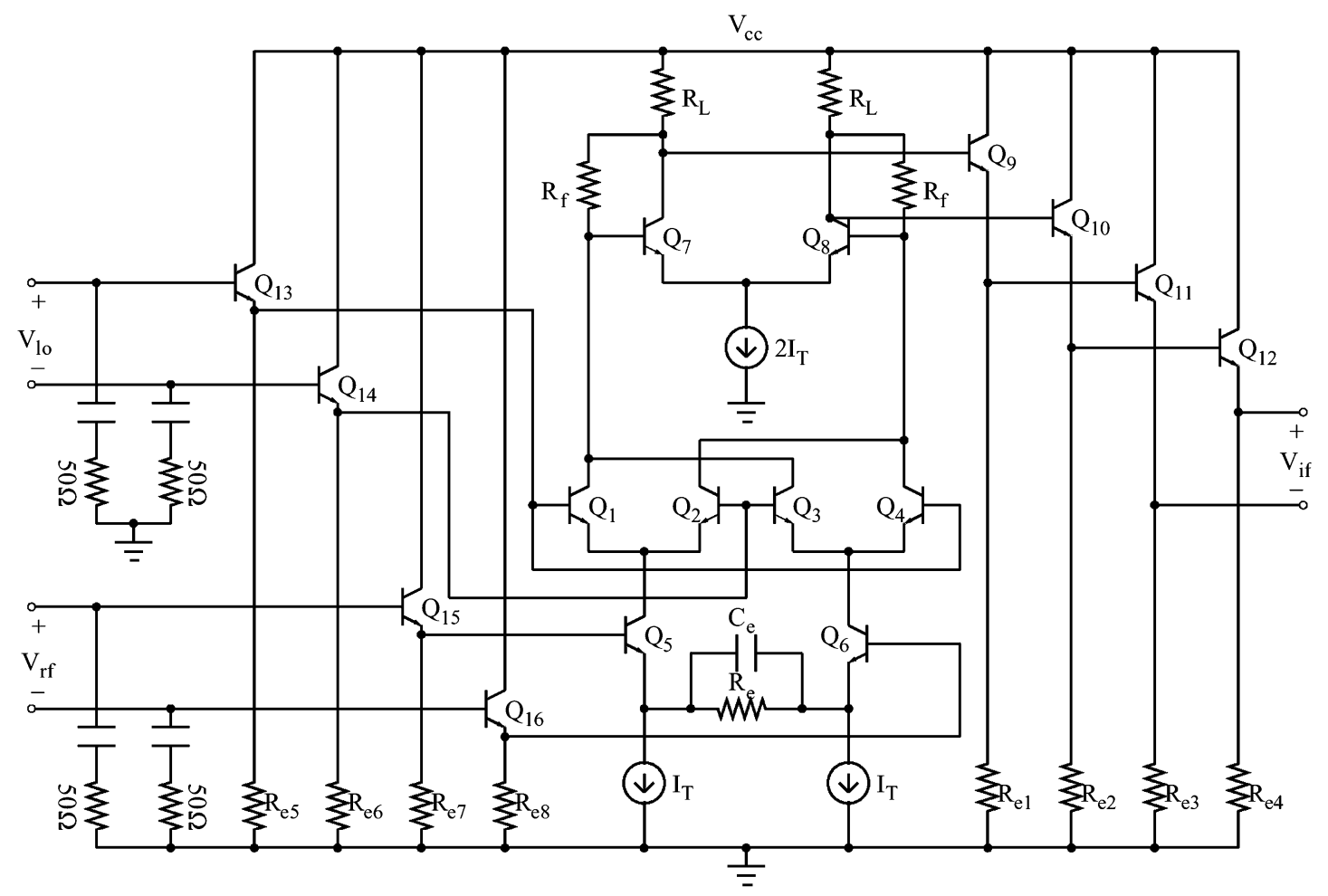

Fig. 6. Modified Gilbert cell mixer.

Including the effect of the shunt feedback load circuit into the expression for the conversion switching gain, (17) results in

$$
\begin{aligned}
& G_{\mathrm{sw}}\left(\omega_{\mathrm{rf}}, \omega_{\mathrm{if}}\right) \\
& =\frac{G_{\mathrm{sw} 0}\left(1-j \frac{\omega_{\mathrm{if}}}{z_{1}}\right)}{\left(1-j \frac{\omega_{\mathrm{if}}}{p_{1}}\right)\left(1+j \omega_{\mathrm{if}} \frac{2 K_{f}}{\omega_{0 f}}-\frac{\omega_{\text {if }}^{2}}{\omega_{0 f}^{2}}\right)\left(1-j \frac{\omega_{\mathrm{rf}}}{p_{3}}\right)}
\end{aligned}
$$

where the low-frequency conversion gain $G_{\mathrm{sw} 0}$ is now determined by the feedback resistance $R_{f}$ as

$$
G_{\mathrm{sw} 0}=\frac{2}{\pi} \frac{R_{f}}{1+\frac{\left(-8+\pi^{2}\right)}{2 \pi^{2}} \frac{I_{T}}{V_{T}} R_{e}}
$$

and the simple pole $p_{2}$ in (17) is transformed into a second-order response with

$$
\begin{aligned}
& \omega_{0 f}=\sqrt{\frac{\left|a_{\mathrm{vo}}\right|}{R_{b 7} C_{\mathrm{cs}} C_{\mathrm{be} 7}\left(R_{s}+2 R_{f}\right)}} \\
& K_{f} \approx \frac{1}{2} \sqrt{\frac{R_{f}+R_{b 7}}{\left|a_{\mathrm{vo}}\right| C_{\mathrm{cs}} R_{b 7}\left(R_{s}+2 R_{f}\right)}}
\end{aligned}
$$

where $\left|a_{\mathrm{vo}}\right|$ is the low-frequency open-loop voltage gain. The details of the analysis of the shunt feedback load circuit are given in the Appendix. The frequency $\omega_{0 f}$ mainly determines the bandwidth of the shunt feedback load circuit. The frequency response peaks when the damping factor becomes $K_{f}<1 / \sqrt{2}$. In general, the following observations regarding conversion gain, bandwidth, and peaking of the shunt feedback load circuit are made.
- The conversion gain is independent of the low-frequency open-loop voltage gain $\left|a_{\mathrm{vo}}\right|$.

- For increasing low-frequency open-loop voltage gain $\left|a_{\mathrm{vo}}\right|$, the bandwidth increases; however, once $K_{f}<1 / \sqrt{2}$, the frequency response peaks, thus, optimal low-frequency open-loop voltage gain exist.

- For increasing feedback resistance $R_{f}$, the conversion gain increases; however, the bandwidth decreases.

Thus, the key to obtaining high conversion gain and wide IF bandwidth without excessive peaking in the Gilbert cell mixer lies in the proper design of the shunt feedback load circuit for optimal performance.

\section{EXPERIMENTAL RESULTS}

The wide-band active mixer design based on the modified Gilbert cell was realized in a $0.8-\mu \mathrm{m} 35-\mathrm{GHz} f_{T}$ SiGe HBT $\mathrm{BiCMOS}$ process. A photograph of the active mixer is shown in Fig. 7. The die size is $0.9 \times 0.9 \mathrm{~mm}^{2}$ and the power consumption is $170 \mathrm{~mW}$ when biased at $+5 \mathrm{~V}$. The active mixer was measured with on-wafer probes and the frequency-dependent cable and connector losses were carefully deembedded from the measurement results.

When both the RF and LO ports are swept in frequency with a fixed IF frequency of $0.4 \mathrm{GHz}$, a conversion gain of $8.5 \mathrm{~dB}$ at $0-\mathrm{dBm} \mathrm{LO}$ power and a 3-dB RF bandwidth of $11 \mathrm{GHz}$ was achieved, as shown in Fig. 8. The LO-IF isolation is better than $-25 \mathrm{~dB}$ despite a single-ended measurement condition for the active mixer.

A 3-dB IF bandwidth of $7.5 \mathrm{GHz}$ was achieved when both the RF and IF port are swept in frequency with the LO fre- 


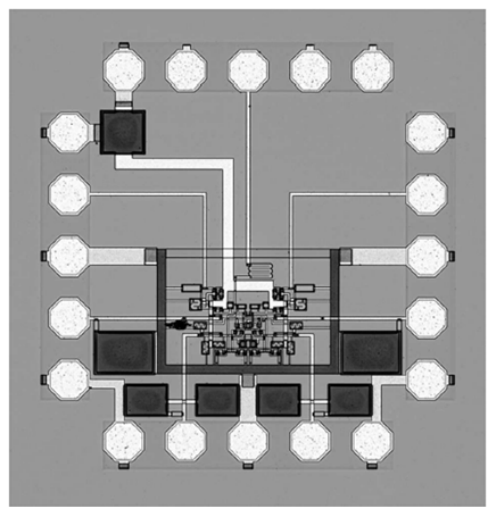

Fig. 7. Photograph of the active mixer.

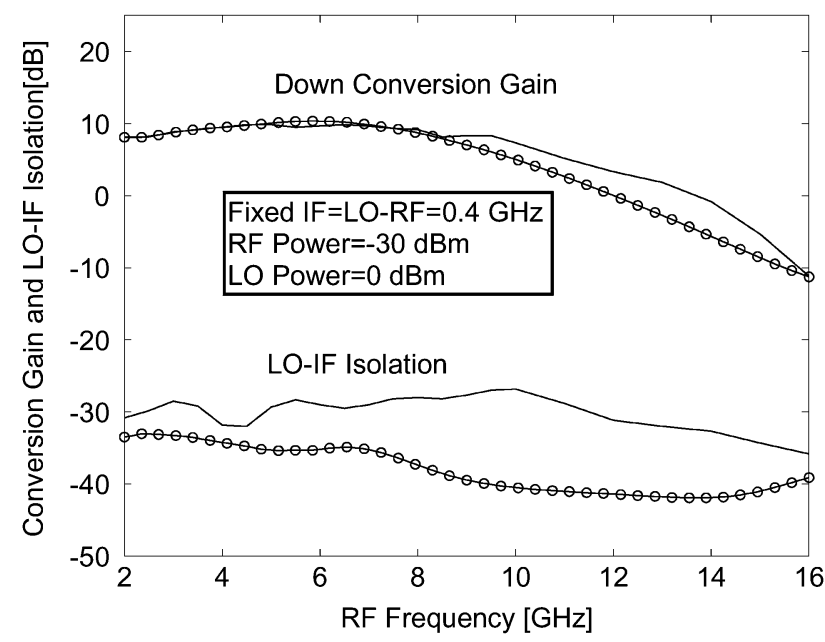

Fig. 8. Measured (-) and simulated (-o-) RF frequency response and LO-IF isolation under single-ended condition.

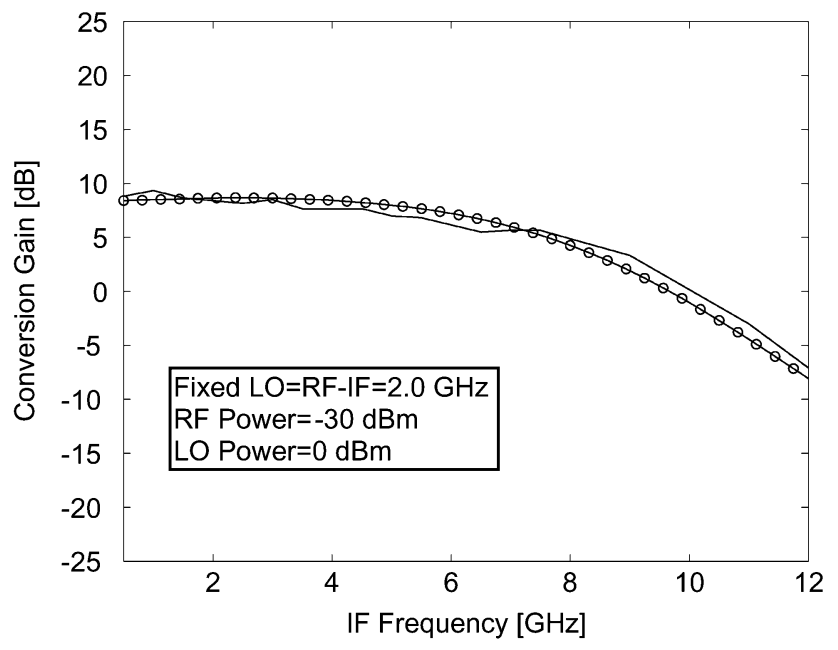

Fig. 9. Measured (-) and simulated (-o-) IF frequency response.

quency fixed at $2 \mathrm{GHz}$, as shown in Fig. 9. The IF bandwidth presents a significant extension compared with the obtainable IF bandwidth from the traditional Gilbert cell, which is limited to approximately $4 \mathrm{GHz}$ for the $0.8-\mu \mathrm{m} 35-\mathrm{GHz} f_{T} \mathrm{SiGe}$ HBT BiCMOS process. An input RF power level of $-18 \mathrm{dBm}$ was measured at 1-dB IF output power compression, as shown

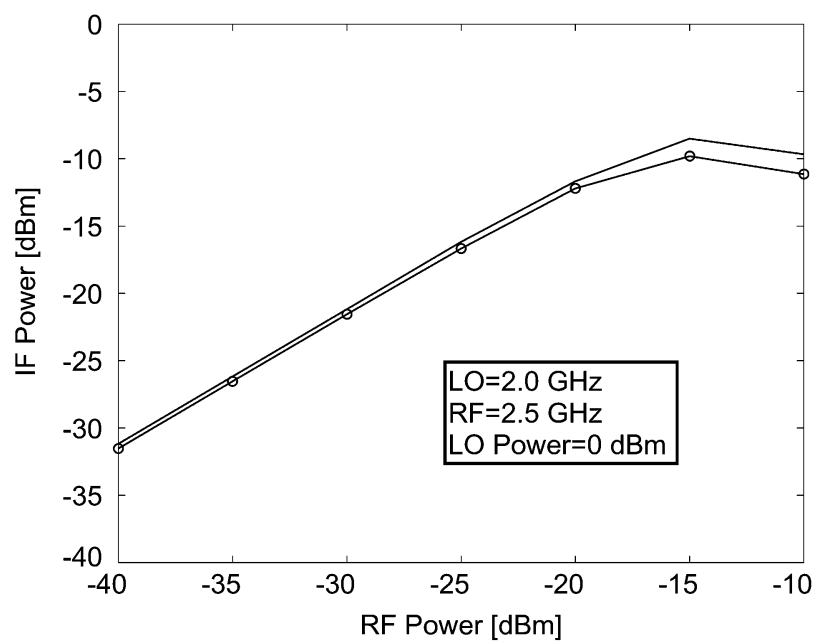

Fig. 10. Measured (-) and simulated (-o-) IF output power versus RF input power.

in Fig. 10. The measured double-sideband noise figure for the wide-band active mixer is around $14 \mathrm{~dB}$.

The measured performance for the active mixer are very well predicted by simulations. The good agreement is a result of accurate high-frequency modeling of SiGe HBT devices and substrate effects associated with interconnection lines and pad structures.

\section{CONCLUSION}

An expression for the conversion gain of SiGe HBT active mixers based on the Gilbert cell topology has been derived using conversion matrix analysis. The expression is capable of predicting the frequency response of the Gilbert cell mixer up to the 3-dB bandwidth and beyond. An optimized active mixer design based on the modified Gilbert cell topology for wide-band operation has been described. The experimental results for a modified Gilbert cell mixer implemented in a $0.8-\mu \mathrm{m} 35-\mathrm{GHz}$ $f_{T}$ SiGe HBT BiCMOS process demonstrated 11- and 7.5-GHz $\mathrm{RF}$ and IF bandwidths, respectively, and a conversion gain of $8.5 \mathrm{~dB}$. This presents a significant extension compared with the theoretically obtainable bandwidths from the traditional Gilbert cell in this process.

\section{APPENDIX \\ ANALYSIS OF SHUNT FEEDBACK LOAD CiRCUIT}

The shunt feedback load circuit in the modified Gilbert cell mixer is most easily analyzed using feedback theory. In order to apply feedback theory, the single-ended equivalent circuit is divided into a basic amplifier and a feedback network, as shown in Fig. 11 [10].

A number of approximations are applied in order to simplify the analysis. First, it is assumed that the signal feedback through the devices is much smaller than the signal feedback through $R_{f}$ so that the base-collector capacitance can be neglected. It is furthermore assumed that the forward transmission through the feedback resistance is negligible compared with the transmission through the devices because the latter has gain. The capacitive loading at the output of the shunt feedback load circuit has 


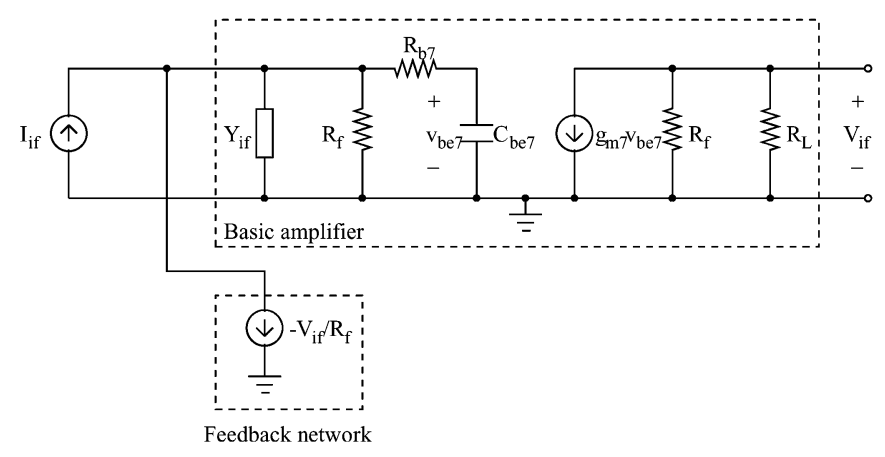

Fig. 11. Single-ended equivalent circuit for the shunt feedback load circuit. The loading due to the feedback network is absorbed in the basic amplifier.

little influence on the frequency response for typical values of load resistances so it is neglected in the analysis. The output of the switching quad at the IF frequency is represented as a Norton equivalent circuit ( $I_{\text {if }}$ and $Y_{\text {if }}$ ) with $Y_{\text {if }}$ absorbed into the basic amplifier. The basic amplifier open loop gain $a$ and feedback network transfer function $f$ are found from Fig. 11 as

$$
a=\frac{-g_{m 7} R_{L}^{\prime} R_{f}}{1+Y_{\text {if }} R_{f}+j \omega_{\text {if }}\left(\left(1+Y_{\text {if }} R_{f}\right) R_{b 7}+R_{f}\right) C_{\mathrm{be} 7}}
$$

and

$$
f=-\frac{1}{R_{f}}
$$

respectively, where $R_{L}^{\prime}=R_{L} R_{f} /\left(R_{L}+R_{f}\right)$ is the effective load resistance when the loading effects from the feedback network is taken into account. The transfer function from the input current $\left(I_{\text {if }}\right)$ to the output voltage at the IF frequency $\left(V_{\text {if }}\right)$ can be represented as

$$
\frac{V_{\text {if }}}{I_{\text {if }}} \approx \frac{-R_{f}}{1+\frac{Y_{\text {if }} R_{f}}{\left|a_{\text {vo }}\right|}+j \omega_{\text {if }} \frac{\left(\left(1+Y_{\text {if }} R_{f}\right) R_{b}+R_{f}\right) C_{\text {be }}}{\left|a_{\text {vo }}\right|}}
$$

where $\left|a_{\mathrm{vo}}\right|=g_{m 7} R_{L}^{\prime}$ is the low-frequency open-loop voltage gain of the basic amplifier. The approximation in (33) is valid for $\left|a_{\mathrm{vo}}\right| \gg 1$. Substituting the Norton admittance

$$
Y_{\mathrm{if}}=\frac{j \omega_{\mathrm{if}} 2 C_{\mathrm{cs}}}{1+j \omega_{\mathrm{if}} C_{\mathrm{cs}} R_{s}}
$$

where the collector resistance has been neglected for simplicity into (33) gives

$$
\begin{aligned}
\frac{V_{\mathrm{if}}}{I_{\mathrm{if}}} & =\frac{-R_{f}\left(1-j \frac{\omega_{\mathrm{if}}}{z_{1 f}}\right)}{1+j \omega_{\mathrm{if}} \frac{2 K_{f}}{\omega_{0 f}}-\frac{\omega_{\mathrm{if}}^{2}}{\omega_{0 f}^{2}}} \\
\frac{1}{z_{1 f}} & =-C_{\mathrm{cs}} R_{s} \\
\omega_{0 f} & =\sqrt{\frac{\left|a_{\mathrm{vo}}\right|}{R_{b 7} C_{\mathrm{cs}} C_{\mathrm{be} 7}\left(R_{s}+2 R_{f}\right)}} \\
K_{f} & \approx \frac{1}{2} \sqrt{\frac{R_{f}+R_{b 7}}{\left|a_{\mathrm{vo}}\right| C_{\mathrm{cs}} R_{b 7}\left(R_{s}+2 R_{f}\right)}}
\end{aligned}
$$

where the last approximation is valid if $C_{\mathrm{cs}}\left(R_{s}\left|a_{\mathrm{vo}}\right|+2 R_{f}\right) \ll$ $C_{\mathrm{be} 7}\left(R_{f}+R_{b 7}\right)$, which is normally the case.

\section{REFERENCES}

[1] B. Gilbert, "A precise four-quadrant multiplier with subnanosecond response," IEEE J. Solid-State Circuits, vol. SC-3, no. 4, pp. 365-373, Dec. 1968.

[2] K. Kobayashi, R. M. Desrosiers, A. Gutuerrez-Aitken, J. C. Cowles, B. Tang, L. T. Tran, T. R. Block, A. K. Oki, and D. C. Streit, "A DC-20 $\mathrm{GHz}$ InP HBT balanced analog multiplier for high-data-rate direct-digital modulation and fiber-optic receiver applications," IEEE Trans. Microw. Theory Tech., vol. 48, no. 2, pp. 194-202, Feb. 2000.

[3] J. Glenn, M. Case, D. Harame, B. Meyerson, and R. Poisson, " $12-\mathrm{GHz}$ Gilbert mixers using a manufacturable $\mathrm{Si} / \mathrm{SiGe}$ epitaxial-base bipolar technology," in IEEE Bipolor/BiCMOS Circuit Tech. Meeting, 1995, pp. $186-189$.

[4] K. Osafune and Y. Yamauchi, "20-GHz 5-dB-gain analog multipliers with AlGaAs/GaAs HBT's," IEEE Trans. Microw. Theory Tech., vol. 42, no. 3, pp. 518-520, Mar. 1994.

[5] C. Campbell and J. Beall, "Design and performance of a highly integrated wide-band active downconverter MMIC," presented at the IEEE Radio Frequency Integrated Circuits Symp., 2001.

[6] S. A. Maas, Nonlinear Microwave and RF Circuits, 2nd ed. Norwood, MA: Artech House, 2003.

[7] C. Pallier, C. Algani, and G. Alquie, "Analysis of conversion gain in a bipolar Gilbert cell microwave mixer," in High Frequency Postgraduate Student Colloq., 1997, pp. 130-135.

[8] K. L. Fong and R. G. Meyer, "Monolithic RF active mixer design," IEEE Trans. Circuits Syst. II, Analog Dig. Signal Process., vol. 46, no. 3, pp. 231-239, Mar. 1999.

[9] R. G. Meyer, "Intermodulation in high-frequency bipolar transistor integrated-circuit mixers," IEEE J. Solid-State Circuits, vol. SC-21, no. 4, pp. 560-563, Apr. 1986.

[10] P. R. Gray and R. G. Meyer, Analysis and Design of Analog Integrated Circuits, 3rd ed. New York: Wiley, 1993.

[11] S. A. Maas, "Theory and analysis of GaAs MESFET mixers," IEEE Trans. Microw. Theory Tech., vol. MTT-32, no. 10, pp. 1402-1406, Oct. 1984.

[12] M. Reisch, High-Frequency Bipolar Transistors. Berlin, Germany: Springer-Verlag, 2003.

[13] J. D. Cressler and G. Niu, Silicon-Germanium Heterojunction Bipolar Transistors, 1st ed. Norwood, MA: Artech House, 2003.

[14] T. K. Johansen, J. Vidkjær, and V. Krozer, "Substrate effects in SiGe HBT modeling," in Proc. Eur. Gallium Arsenide and Other Compound Semiconductors Application Symp., Munich, Germany, Oct. 2003, pp. 879-882.

[15] K. Ohhata, E. Ohue, and K. Washio, "Design of a 32.7-GHz bandwidth AGC amplifier IC with wide dynamic range implemented in SiGe HBT," IEEE J. Solid-State Circuits, vol. 34, no. 9, pp. 1290-1297, Sep. 1999.

[16] E. M. Cherry and D. E. Hooper, "The design of wide-band transistor feedback amplifiers," Proc. Inst. Elect. Eng., vol. 110, no. 2, pp. 375-389, Feb. 1963.

[17] T. K. Johansen, "Monolithic microwave integrated circuits for wideband SAR system," Ph.D. dissertation, Dept. Elect. Eng., Tech. Univ. Denmark, Lyngby, Denmark, 2003. 


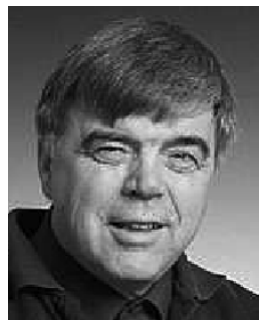

Jens Vidkjær (S'72-M'72) received the M.S. and Ph.D. degrees from the Technical University of Denmark, Lyngby, Denmark, in 1968 and 1975, respectively.

Since 1970, he has been with the Electronics Laboratory and the Semiconductor Laboratory, Technical University of Denmark, where he is currently a Reader with the Electromagnetic System Group, Ørsted॰DTU. His research areas have included RF power-amplifier design, computer-aided design (CAD) methods, device modeling, measurement accuracies, and MMIC design

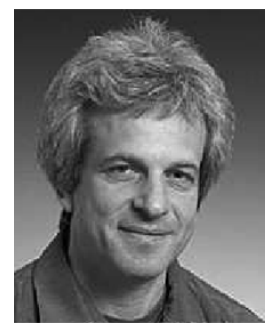

Viktor Krozer (M-93-SM'03) was born in Leningrad, Russia, in 1958. He received the Dipl.-Ing. degree in electrical engineering and Dr.-Ing. degree (suma cum laude) from the Technische Hochschule Darmstadt (TH Darmstadt), Darmstadt, Germany, in 1984 and 1991, respectively. His Dr.-Ing. thesis focused on the development of analysis methods for large-signal nonlinear microwave circuits and semiconductor device simulation.

In 1991, he joined the Microwave Electronics Laboratory, TH Darmstadt, as a Senior Research Scientist involved in the areas of high-temperature semiconductor device operation and submillimeter-wave device development. From 1996 to 2002, he was a Professor of electrical engineering with the Technical University of Chemnitz (TU Chemnitz), where he was involved in the area of microwave electronics, and where he was the Head of the Microwave Laboratory. Since 2002, he has been with the Section of Electromagnetic Systems, Ørsted•DTU, Lyngby, Denmark. He has contributed to several book chapters and has authored or coauthored over 100 papers appearing in international journals and conferences. His research interests include physical modeling of semiconductor devices and circuits, MMIC and multichip module (MCM) technology, reliability of semiconductor devices, and submillimeter-wave devices and systems. 